\title{
Improvement of endothelial function in patients with hypertension and type 2 diabetes after treatment with telmisartan
}

\author{
Takehiko Wago, Takanobu Yoshimoto, Itaru Akaza, Kyoichiro Tsuchiya, Hajime Izumiyama, Masaru Doi \\ and Yukio Hirata
}

Telmisartan, a selective antagonist for angiotensin type1 receptor and a partial agonist for peroxisome proliferator-activated receptor- $\gamma$, decreases blood pressure and has been shown to improve glucose and lipid metabolism, suggesting potential cardiovascular protective effects. In this study, we investigated whether long-term treatment with telmisartan improved endothelial function in 35 hypertensive patients with type 2 diabetes mellitus (T2DM). Office and home early morning blood pressure levels and flow-mediated vasodilation (FMD) were evaluated before and after 12 months of treatment with telmisartan. Blood samples were also obtained for measurement of several biochemical parameters and of adiponectin (AN) and highly sensitive C-reactive protein (hs-CRP) before and after treatment. After 12 months of treatment, office and morning blood pressure levels had significantly decreased, and levels of plasma glucose, glycosylated hemoglobin, total cholesterol, triglyceride and low-density lipoprotein cholesterol had also significantly decreased. Plasma AN and high-density lipoprotein cholesterol levels increased, but hs-CRP levels decreased. Furthermore, FMD significantly increased; changes in percent FMD showed a significant negative correlation with changes in systolic and diastolic blood pressure and a significant positive correlation with changes in AN. Stepwise multivariate regression analysis revealed that changes in plasma AN and office systolic blood pressure were both independent determinants for endothelial function after telmisartan treatment. In conclusion, this study shows that long-term treatment with telmisartan improves not only blood pressure and glucose and lipid metabolism but also endothelial function in hypertensive patients with T2DM, possibly by increased circulating AN and decreased blood pressure.

Hypertension Research (2010) 33, 796-801; doi:10.1038/hr.2010.107; published online 17 June 2010

Keywords: adiponectin; endothelial function; flow-mediated vasodilation; telmisartan

\section{INTRODUCTION}

Essential hypertension and type 2 diabetes mellitus (T2DM), as well as dyslipidemia, smoking and certain genetic factors, are major risk factors for atherosclerosis and are associated with a high incidence of cardiovascular events. Endothelial dysfunction, characterized by impaired endothelium-dependent vasodilation, is considered to be an early event in the development of atherosclerosis. ${ }^{1}$ A growing body of evidence suggests that endothelial dysfunction is reversible after treatment and may be a prognostic predictor for improvement in cardiovascular disease. ${ }^{2-4}$ In hypertensive and diabetic conditions, both the renin-angiotensin system and insulin resistance are believed to have important roles in the pathogenesis of endothelial dysfunction and in the development of atherosclerosis. ${ }^{5-8}$ On the basis of their cardiovascular protective effects, pharmacological blockers of the renin-angiotensin system such as angiotensin-converting enzyme inhibitors (ACEI) and angiotensin II receptor blockers (ARB) have been widely used to treat patients with hypertension, whereas insulin sensitizers such as peroxisome proliferator-activated receptor (PPAR)- $\gamma$ agonists have been used to treat patients with hypertension and diabetes, respectively. $3,8,9$

Among several ARBs used clinically worldwide, telmisartan is a unique compound because it functions as a partial agonist for PPAR $\gamma .{ }^{10-13}$ In hypertensive patients associated with T2DM and/or the metabolic syndrome, several clinical studies have shown that telmisartan has beneficial effects on glucose and lipid metabolism in addition to its antihypertensive effect. ${ }^{14-17}$ Thus, cardiovascular protective effects of telmisartan in hypertensive patients with T2DM could be due to the combined actions of its potent blockade in the renin-angiotensin system and its partial activation of PPAR $\gamma$.

In this study, we investigated whether long-term treatment with telmisartan improved endothelial function in patients with essential hypertension and T2DM, and if so, we sought to clarify the determinant factor(s) responsible for the improvement. 


\section{METHODS}

Study population and design

Patient demographics are shown in Table 1. In all, 35 patients with T2DM and hypertension were recruited. Regardless of whether patients were taking antihypertensive or antidiabetic drugs, hypertension was defined as elevated systolic blood pressure (SBP) $\geqslant 135 \mathrm{~mm} \mathrm{Hg}$ and/or elevated diastolic blood pressure (DBP) $\geqslant 85 \mathrm{~mm} \mathrm{Hg}$ measurements taken at home in the morning, and T2DM was defined as elevated fasting glucose $\left(\geqslant 126 \mathrm{mg} \mathrm{dl}^{-1}\right)$ and elevated glycosylated hemoglobin $(\geqslant 6.5 \%)$. A total of 17 patients were current smokers, and 15 had dyslipidemia. Diabetic complications, previous cardiovascular events and all antihypertensive, antidiabetic and cholesterol-lowering drugs used are summarized in Table 1.

This was a 1-year, open-label prospective study. The study protocol was approved by the Ethics Committee of our institute, and informed consent was obtained from each patient. Each patient's prestudy medications were maintained throughout the study period, except for ACEIs and ARBs (if used), which were discontinued before the entry of participants into the study and replaced with telmisartan. All patients were started on telmisartan $(40 \mathrm{mg})$ and maintained on the same dose throughout the study period without any adverse effects. Measurements of physiological and biochemical parameters were evaluated before and after the 12-month-long treatment.

\section{Measurement of morning (home) blood pressure}

Morning blood pressure was measured at home using an electronic oscillometric arm-cuff device (HEM-075; Omron Healthcare, Tokyo, Japan) according to the Japanese Society of Hypertension Guidelines for Self-Monitoring Blood Pressure at Home. ${ }^{18}$ Briefly, after 5 min of rest in the sitting position, patients carried out three successive measurements of blood pressure in the time between waking and 1000 hours (within $1 \mathrm{~h}$ of rising, after urination and before breakfast and medication). The mean blood pressure of 3 consecutive days was taken as the morning blood pressure value. The morning blood

Table 1 Baseline demographic characteristics of the patients studied

\begin{tabular}{lc}
\hline Age (years) & $61.5 \pm 1.8$ \\
Sex (male or female) & $21 / 14$ \\
Smoker & $17(48.5 \%)$ \\
Dyslipidemia & $15(42.9 \%)$ \\
Serum creatinine $\left(\mathrm{mgdl}^{-1}\right)$ & $0.83 \pm 0.57$ \\
& \\
Diabetic complications & \\
$\quad$ Proliferative retinopathy & $14(40 \%)$ \\
Proteinuria & $14(40 \%)$ \\
Neuropathy & $18(51.4 \%)$ \\
& \\
Previous cardiovascular events & \\
Coronary artery disease & \\
Stroke & $5(14.3 \%)$ \\
Peripheral arterial disease & $3(8.6 \%)$ \\
& $3(8.6 \%)$ \\
Medications & \\
Previous ACEl or ARB & \\
Calcium channel blocker & \\
$\alpha-B l o c k e r$ & $15(42.9 \%)$ \\
$\beta$-Blocker & $10(28.6 \%)$ \\
Diuretics & $2(5.7 \%)$ \\
Statin & $2(5.7 \%)$ \\
Sulfonylurea & $3(8.6 \%)$ \\
Biguanide & $10(28.6 \%)$ \\
Thiazolidine & $6(17.2 \%)$ \\
$\alpha$-Glucosidase inhibitor & $2(5.7 \%)$ \\
Insulin & $3(8.6 \%)$ \\
\hline
\end{tabular}

Abbreviations: ACEI, angiotensin-converting enzyme inhibitor; ARB, angiotensin type 1 receptor antagonist.

Values are number of patients or mean \pm s.d. Percentage (\%) are indicated in parenthesis. pressure values before and after treatment were determined as the average value of each of 3 consecutive days.

\section{Measurement of vascular endothelial function and office blood pressure}

To assess vascular endothelial function, flow-mediated vasodilation (FMD) was measured by ultrasound, using a SONOS 5500 machine (Philips Medical System, Andover, MA, USA) equipped with an $11 \mathrm{Mhz}$ ultrasound probe as described previously. ${ }^{19,20}$ Office blood pressure was defined as blood pressure measured by a mercury column sphygmomanometer after a seated, $5 \mathrm{~min}$ rest and before FMD measurement. The percent FMD (\%FMD) of the brachial artery was expressed as the percentage increase in maximal diameter from the baseline within $120 \mathrm{~s}$ after cuff deflation. Inter- and intra-observer variability rates for FMD in our laboratory were previously shown to be $8.3 \pm 2.1 \%$ and $6.9 \pm 1.3 \%$, respectively. ${ }^{19}$

\section{Measurements of biochemical parameters}

Blood samples were drawn from the antecubital vein after an overnight fast lasting at least $12 \mathrm{~h}$. Fasting plasma glucose, glycosylated hemoglobin, serum total cholesterol, serum triglyceride, low-density lipoprotein cholesterol and high-density lipoprotein cholesterol levels were measured by an autoanalyzer (HLC-723G7; Tosoh Corporation, Tokyo, Japan). Fasting immunoreactive insulin was measured by an immunoradiometric assay (Mitsubishi Chemical Medience, Tokyo, Japan). Plasma total adiponectin (AN) and highly sensitive C-reactive protein (hs-CRP) levels were measured by enzyme-linked immunosorbent assays (Fujirebio, Tokyo, Japan) and latex-enhanced immunometric assays (Mitsubishi Chemical Medience), respectively.

\section{Statistical analysis}

Results are presented as mean \pm standard deviation (s.d.). Changes in various parameters after 1 year of treatment with telmisartan were analyzed using the paired Student's $t$-test. Simple linear regression analysis was used for dependent factors that were independently associated with a dependent variable. A forward stepwise multivariate regression analysis was used to identify factors that were independently associated with a dependent variable. Independent variables included in this model were those that reached statistical significance in simple linear regression analysis.

$P$-values $<0.05$ were considered to be statistically significant. All statistical analyses were carried out using Windows software StatView 5.0 (SAS Institute, Cary, NC, USA).

\section{RESULTS}

Changes in blood pressure, vascular endothelial function and biochemical and metabolic parameters after treatment with telmisartan

Table 2 shows the changes in the various parameters examined before and after treatment. Morning and office blood pressure (SBP and DBP) levels were significantly decreased $(P<0.001)$ after 1 year of treatment with telmisartan; a significant decrease in body mass index was also noted. Percentage FMD was also significantly increased after treatment, suggesting improved vascular endothelial function after treatment. Fasting plasma glucose, glycosylated hemoglobin, total cholesterol, triglyceride, low-density lipoprotein cholesterol and hsCRP levels, but not fasting immunoreactive insulin, decreased significantly $(P<0.05)$, whereas total AN and high-density lipoprotein cholesterol levels were significantly increased $(P<0.001)$ after treatment. As shown in Table 1, 15 (42.9\%) and $3(8.3 \%)$ patients had been taking ACEI/ARB or thiazolidinedione before this study, respectively. There were no significant differences in blood pressure changes between those patients with and without pretreatment with ARB/ ACEI after telmisartan treatment (data not shown). There were no significant differences in changes in metabolic markers and plasma AN levels between those patients with and without pretreatment with pioglitazone after telmisartan treatment (data not shown). 
Table 2 Effects of treatment with telmisartan on blood pressure, endothelial function, biochemical, and metabolic parameters

\begin{tabular}{|c|c|c|c|}
\hline Variables & Before treatment & After treatment & P-value \\
\hline Morning SBP(mm Hg) & $151 \pm 17$ & $126 \pm 8$ & $P<0.001$ \\
\hline Morning DBP(mm Hg) & $89 \pm 10$ & $73 \pm 6$ & $P<0.001$ \\
\hline Office SBP(mm Hg) & $153 \pm 13$ & $138 \pm 12$ & $P<0.001$ \\
\hline Office DBP(mm Hg) & $89 \pm 7$ & $77 \pm 6$ & $P<0.001$ \\
\hline$\% F M D(\%)$ & $4.0 \pm 2.2$ & $5.9 \pm 2.2$ & $P<0.001$ \\
\hline BMI $\left(\mathrm{kg} \mathrm{m}^{-2}\right)$ & $26.0 \pm 4.4$ & $24.2 \pm 2.9$ & $P=0.027$ \\
\hline FPG (mg dl-1) & $199 \pm 58$ & $140 \pm 51$ & $P<0.001$ \\
\hline $\mathrm{FIRI}\left(\mu \cup \mathrm{ml}^{-1}\right)$ & $17.8 \pm 14.6$ & $24.3 \pm 20.5$ & $P=0.221$ \\
\hline $\mathrm{HbAlc}(\%)$ & $8.7 \pm 1.5$ & $7.4 \pm 1.2$ & $P<0.001$ \\
\hline TC $\left(\mathrm{mg} \mathrm{dl}^{-1}\right)$ & $202 \pm 36$ & $181 \pm 27$ & $P=0.038$ \\
\hline $\mathrm{TG}\left(\mathrm{mg} \mathrm{dl}^{-1}\right)$ & $159 \pm 87$ & $117 \pm 47$ & $P=0.011$ \\
\hline LDL-C (mg dl-1) & $119 \pm 28$ & $101 \pm 24$ & $P<0.001$ \\
\hline HDL-C (mg dl-1) & $52 \pm 15$ & $63 \pm 20$ & $P<0.001$ \\
\hline Adiponectin $\left(\mu \mathrm{g} \mathrm{ml}^{-1}\right)$ & $5.6 \pm 2.7$ & $8.0 \pm 4.0$ & $P<0.001$ \\
\hline hs-CRP (mg dl-1) & $0.13 \pm 0.16$ & $0.07 \pm 0.10$ & $P=0.011$ \\
\hline
\end{tabular}

Abbreviations: BMI, body mass index; DBP, diastolic blood pressure; FIRI, fasting immunoreactive insulin; \%FMD, percent flow-mediated vasodilation; FPG, fasting plasma glucose; HbAlc, hemoglobin Alc; HDL-C, high-density lipoprotein cholesterol; hs-CRP, highly sensitive C-reactive protein; LDL-C, low-density lipoprotein cholesterol; SBP, systolic blood pressure; TC, total cholesterol; TG, triglyceride.

\section{Correlations of FMD with blood pressure and biochemical metabolic parameters}

To determine which parameters were responsible for the improvement in endothelial function after telmisartan treatment, a simple regression analysis of the changes in \%FMD and other parameters was carried out. As shown in Figure 1 and Table 3, the changes in \%FMD were negatively correlated with changes in morning and office blood pressure (SBP and DBP) levels and positively correlated with changes in $\mathrm{AN}$; however, changes in \%FMD and other biochemical parameters were not correlated (Table 3). There was a significant positive correlation between \%FMD and absolute value of total AN after treatment $(r=0.44, P=0.017)$, whereas no significant correlation was present before treatment $(r=0.069, P=0.71)$.

\section{Changes in office SBP and AN are independent determinants of endothelial function}

Because the changes in morning and office blood pressure (SBP and DBP) levels and AN were significantly correlated with changes in $\%$ FMD in a simple linear regression analysis, these factors were included in a stepwise multivariate regression analysis as possible independent variables. As shown in Table 4, changes in office SBP ( $\beta=0.43, P=0.012)$ and $\mathrm{AN}(\beta=-0.38, P=0.027)$ were found to be independent determinants for endothelial function after long-term telmisartan treatment.

\section{DISCUSSION}

This study clearly showed that long-term treatment with telmisartan not only reduced blood pressure but also improved metabolic parameters and endothelial function in hypertensive patients with T2DM. We found that an increase in FMD correlated with decreased blood pressure and increased plasma AN levels and that both office SBP and plasma AN levels are independent determinants for improvement of FMD. These results suggest that blood pressure and AN are both key factors responsible for the improvement in endothelial function after long-term telmisartan treatment.

After 1 year of treatment with telmisartan in this study, morning and office blood pressure levels, as well as body mass index, decreased significantly, and glycemic control and lipid profiles improved, suggesting that improved endothelial function after long-term telmisartan treatment is associated with a reduction in blood pressure and improvement in several biochemical metabolic parameters. Telmisartan, in addition to its blood pressure-lowering effect, has been reported to exert its antiatherogenic effect by blocking angiotensin II-induced oxidative stress and inflammation in cardiovascular tissues. $^{3,9,21}$ Furthermore, telmisartan has been reported to have an antidiabetic effect, ${ }^{14-17}$ possibly by improving insulin sensitivity. Telmisartan, a unique ARB, has proven to be a partial PPAR $\gamma$ agonist because of its structural similarity to thiazolidinedione derivatives, thereby improving insulin sensitivity and reducing inflammation. ${ }^{10,11,22}$ Indeed, several previous studies showed that, compared with other ARBs, telmisartan has more beneficial effects on metabolic and endothelial function in hypertensive patients with the metabolic syndrome, IGT or T2DM, ${ }^{23-25}$ suggesting a PPAR $\gamma$ agonistic action in addition to its function as an ARB. In the recent ONTARGET study, however, telmisartan was shown to be as effective as the ACEI ramipril in reducing the frequency of newly diagnosed diabetes in patients with coronary, peripheral and cerebrovascular disease without diabetes. ${ }^{26}$ It is possible that the collective beneficial effects of telmisartan on metabolic parameters (fasting plasma glucose, glycosylated hemoglobin, total cholesterol, triglyceride, low-density lipoprotein cholesterol, high-density lipoprotein cholesterol) and an inflammation marker (hs-CRP), as shown in this study, are largely attributable to angiotensin II blockade, but they may also be partly due to PPAR $\gamma$ agonist action.

This study further showed that endothelial function significantly improved after long-term treatment with telmisartan in hypertensive patients with T2DM. Our results are in agreement with those of previous studies showing that short-term (4-12 weeks) treatment with telmisartan improved endothelial function in patients with essential hypertension and diabetes. ${ }^{24,27,28}$ In addition to the previous shortterm observations, ${ }^{24,27,28}$ we clearly showed the markedly beneficial effect on endothelial function after long-term (1-year) treatment with telmisartan. Furthermore, our study showed for the first time that the improvement in FMD correlated with the reduction in blood pressure and the increase in circulating AN after treatment with telmisartan. However, it may be difficult to determine whether the improvement in endothelial function after long-term telmisartan treatment should be attributed to ARB action, PPAR $\gamma$ agonist action, or both. It is noteworthy that telmisartan has been shown to improve endothelial function to a greater degree than losartan in hypertensive patients with impaired glucose tolerance, ${ }^{24}$ suggesting the possible role of telmisar$\tan$ as a partial PPAR $\gamma$ agonist in endothelial function.

This study revealed that the increase in AN levels after long-term treatment with telmisartan is an independent determinant for the improvement of endothelial function. In addition to the correlation between changes in AN and \%FMD, the absolute level of total AN was significantly correlated with \%FMD after telmisartan treatment. As our findings confirmed, several previous studies showed that treatment with telmisartan increased plasma AN levels in patients with T2DM ${ }^{29-31}$ whereas treatment with ACEIs (ramipril) or other ARBs (losartan, candesartan, valsartan) did not affect AN levels. ${ }^{30,31}$ Thiazolidinedione derivatives have been shown to increase the expression of $\mathrm{AN},{ }^{32}$ a collagen-like protein produced specifically by adipocytes and secreted into circulation. Circulating AN levels have been reported to be lower in patients with morbid obesity, metabolic syndrome or T2DM. ${ }^{33}$ We recently showed that short-term (12-week) treatment with pioglitazone significantly increased plasma AN levels in patients with metabolic syndrome; this correlated with improved endothelial 

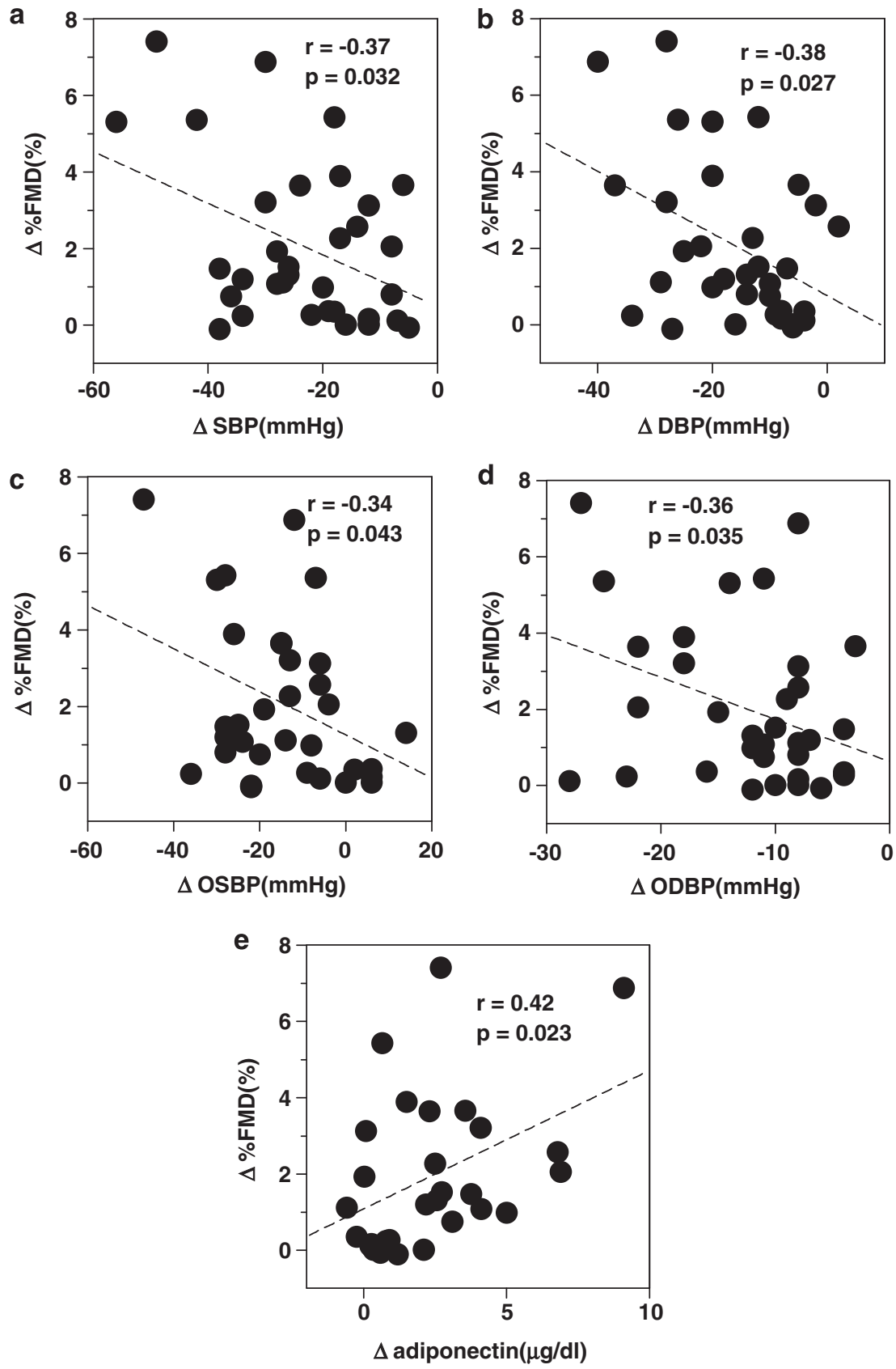

Figure 1 Correlations between endothelial function and blood pressure or plasma AN by a simple regression analysis. Correlations between changes in $\% \mathrm{FMD}(\triangle \% \mathrm{FMD})$ and changes in (a) morning SBP $(\triangle \mathrm{MSBP}),(\mathbf{b})$ morning DBP $(\triangle \mathrm{MDBP})$, (c) office systolic blood pressure $(\triangle \mathrm{OSBP}),(\mathbf{d})$ office DBP $(\triangle \mathrm{ODBP})$ and (e) plasma $\mathrm{AN}(\triangle \mathrm{AN})$ are shown. Broken lines indicate regression lines.

function. ${ }^{34}$ Furthermore, it has been shown that plasma AN level is predictive for endothelial function in healthy participants ${ }^{35}$ and that plasma levels of the high-molecular-weight form of AN are a more biologically active marker than levels of total AN in humans. ${ }^{35,36}$ In fact, it has been shown that AN directly stimulates nitric oxide production in endothelial cells in vitro. ${ }^{37}$ Taking these findings together, it is possible that even the mild change in total plasma AN level after telmisartan treatment observed in this study may be involved in improving endothelial function by increased endothelial nitric oxide production.

Although it is well recognized that hypertension is associated with endothelial dysfunction, it remains unproven whether antihyperten- sive drugs improve endothelial function solely by decreasing blood pressure. ${ }^{38}$ Nevertheless, the present study clearly revealed that the decrease in office SBP by telmisartan treatment was another independent determinant for improved endothelial function. In contrast to our study, Perl et al. ${ }^{24}$ reported that the improvement in FMD by telmisartan was independent of blood pressure. The discrepancy between Perl's study and ours may be attributable to the different study populations and designs. The durations of treatment in our study and Perl's study were 1 year and 12 weeks, ${ }^{24}$ respectively. In addition, the patients enrolled in our study had higher blood pressure and showed a more profound antihypertensive effect by telmisartan than those in Perl's study. ${ }^{24}$ This suggests that the longer period of 
Table 3 Correlations of the changes $(\Delta)$ of \%FMD and those of blood pressure, biochemical, and metabolic parameters after treatment with telmisartan

\begin{tabular}{lrl}
\hline Variables & $r$ & P-value \\
\hline$\Delta$ Morning SBP & -0.37 & 0.032 \\
$\Delta$ Morning DBP & -0.38 & 0.027 \\
$\Delta$ Office SBP & -0.35 & 0.043 \\
$\Delta$ Office DBP & -0.36 & 0.035 \\
$\Delta$ BMI & 0.21 & 0.52 \\
$\Delta$ FPG & -0.16 & 0.44 \\
$\Delta$ FIRI & 0.06 & 0.83 \\
$\Delta$ HbA1c & -0.05 & 0.83 \\
$\Delta$ TC & -0.07 & 0.75 \\
$\Delta$ TG & -0.08 & 0.72 \\
$\Delta$ LDL-C & 0.08 & 0.68 \\
$\Delta$ HDL-C & 0.11 & 0.59 \\
$\Delta$ Adiponecitn & 0.42 & 0.023 \\
$\Delta$ hs-CRP & 0.02 & 0.93 \\
\hline Abrevalin: BML &
\end{tabular}

Abbreviations: BMI, body mass index; DBP, diastolic blood pressure; FIRI, fasting immunoreactive insulin; \%FMD, percent flow-mediated vasodilation; FPG, fasting plasma glucose; HbAlc, hemoglobin-Alc; HDL-C, high-density lipoprotein cholesterol; hs-CRP, highly sensitive C-reactive protein; LDL-C, low-density lipoprotein cholesterol; SBP, systolic blood pressure; TC, total cholesterol; TG, tri-glyceride

The $r$ value represents the coefficient of Pearson's correlation.

Table 4 Forward stepwise multivariate analysis of determinant for $\Delta \% \mathrm{FMD}$ after treatment with telmisartan

\begin{tabular}{lrc}
\hline Variables & $\beta$ & P-value \\
\hline$\Delta$ Adiponectin & 0.43 & 0.012 \\
$\Delta$ Office SBP & -0.38 & 0.027
\end{tabular}

Abbreviations: \% FMD, percent flow-mediated vasodilation; SBP, systolic blood pressure. $R^{2}=0.21, F=6.2$, and $P=0.006$.

$R^{2}$ represents coefficient of determination for the model. $\beta$ represents standard regression coefficient.

$\Delta \%$ FMD as a dependent variable, and $\Delta$ adiponectin and $\Delta$ office SBP as independent variables.

telmisartan treatment with a greater antihypertensive effect may have contributed to the significant improvement in endothelial function in hypertensive patients with T2DM.

A multivariate analysis in this study showed that changes in both office SBP and AN are independent determinants of FMD improvement after telmisartan treatment. However, it remains unknown why only office SBP among several blood pressure measurements was found to be a determinant for improvement in FMD after telmisartan treatment. This may be partly accounted for by the small size of the study population and/or the relatively accurate values of office blood pressure measured just before FMD measurement.

Our results seem to be in accordance with those of Perl's study that showed that improvement in FMD after treatment with telmisartan was independent of glucose metabolism. ${ }^{24}$ However, it remains unknown why treatment of diabetes and dyslipidemia, both major cardiovascular risk factors, did not affect endothelial function in this study. It is tempting to speculate that the anti-inflammatory and antiatherosclerotic effects of telmisartan may contribute to the improvement of endothelial function by increased AN and decreased oxidative stress. $^{28}$

There are several limitations in this study. First, it is an open-label study that did not use a placebo or specify other antihypertensive drugs for comparison. Therefore, it seems difficult to determine the precise mechanism(s) by which telmisartan functions as an ARB and/ or PPAR $\gamma$ agonist to improve endothelial function after the year-long treatment observed here. Second, because improved endothelial function was shown in patients with hypertension after treatment with various antihypertensive agents, ${ }^{38-40}$ we could not exclude the possibility that some patients pretreated with various antihypertensive drugs already had improved endothelial function before this study. Nevertheless, this work clearly shows that endothelial function was significantly and similarly improved after 1 year of treatment with telmisartan either with or without pretreatment with ACEI/ARB and/ or thiazolidinedione before the entry of patients into this study. This suggests that newly started telmisartan could have contributed to the improvement in endothelial function observed in this study. Finally, all our patients followed the standard management protocols of diet and mild exercise for diabetes used at our department. As this was an open-label study (a limitation, as discussed), it is difficult to differentiate the influence of telmisartan from the effects of our standard diabetes management. Nevertheless, several clinical studies that included control subjects have already shown that treatment with telmisartan led to significant improvements in metabolic profile and body weight. ${ }^{14-17,41}$ Thus, it is reasonable to assume that the improvement in endothelial function observed in our study could have resulted from telmisartan treatment.

In conclusion, our study shows that 1-year treatment with telmisartan improved not only blood pressure and glucose and lipid metabolism but also endothelial function in hypertensive patients with T2DM, and that increases in plasma AN levels and decreases in office SBP are both independent determinants for improvement in endothelial function. These findings suggest a therapeutic role for telmisartan in improving endothelial function for the prevention of atherosclerosis in hypertensive patients with T2DM.

\section{ACKNOWLEDGEMENTS}

This study was supported in part by Grants-in-Aid from the Ministry of Education, Science, Sports, Culture and Technology, and by the Ministry of Health, Labor and Welfare of Japan

1 Deanfield JE, Halcox JP, Rabelink TJ. Endothelial function and dysfunction: testing and clinical relevance. Circulation 2007; 115: 1285-1295.

2 Gokce N, Keaney Jr JF, Hunter LM, Watkins MT, Nedeljkovic ZS, Menzoian JO, Vita JA. Predictive value of noninvasively determined endothelial dysfunction for long-term cardiovascular events in patients with peripheral vascular disease. J Am Coll Cardiol 2003; 41: 1769-1775

3 Mancini GB, Henry GC, Macaya C, O'Neill BJ, Pucillo AL, Carere RG, Wargovich TJ, Mudra H, Luscher TF, Klibaner MI, Haber HE, Uprichard AC, Pepine CJ, Pitt B. Angiotensin-converting enzyme inhibition with quinapril improves endothelial vasomotor dysfunction in patients with coronary artery disease. The TREND (Trial on Reversing ENdothelial Dysfunction) Study. Circulation 1996; 94: 258-265.

4 Treasure CB, Klein JL, Weintraub WS, Talley JD, Stillabower ME, Kosinski AS, Zhang J, Boccuzzi SJ, Cedarholm JC, Alexander RW. Beneficial effects of cholesterol-lowering therapy on the coronary endothelium in patients with coronary artery disease. $N$ Engl J Med 1995; 332: 481-487.

5 Cooper SA, Whaley-Connell A, Habibi J, Wei Y, Lastra G, Manrique C, Stas S, Sowers JR. Renin-angiotensin-aldosterone system and oxidative stress in cardiovascular insulin resistance. Am J Physiol Heart Circ Physiol 2007; 293: H2009-H2023.

6 Ferrario CM, Strawn WB. Role of the renin-angiotensin-aldosterone system and proinflammatory mediators in cardiovascular disease. Am J Cardiol 2006; 98: 121-128.

7 Rask-Madsen C, King GL. Mechanisms of disease: endothelial dysfunction in insulin resistance and diabetes. Nat Clin Pract Endocrinol Metab 2007; 3: 46-56.

8 Pistrosch F, Passauer J, Fischer S, Fuecker K, Hanefeld M, Gross P. In type 2 diabetes, rosiglitazone therapy for insulin resistance ameliorates endothelial dysfunction independent of glucose control. Diabetes Care 2004; 27: 484-490.

9 Yao EH, Fukuda N, Matsumoto T, Kobayashi N, Katakawa M, Yamamoto C, Tsunemi A, Suzuki R, Ueno T, Matsumoto K. Losartan improves the impaired function of endothelial progenitor cells in hypertension via an antioxidant effect. Hypertens Res 2007; 30: 1119-1128.

10 Benson SC, Pershadsingh HA, Ho Cl, Chittiboyina A, Desai P, Pravenec M, Qi N, Wang J, Avery MA, Kurtz TW. Identification of telmisartan as a unique angiotensin II receptor 
antagonist with selective PPARgamma-modulating activity. Hypertension 2004; 43: 993-1002.

11 Janke J, Schupp M, Engeli S, Gorzelniak K, Boschmann M, Sauma L, Nystrom FH, Jordan J, Luft FC, Sharma AM. Angiotensin type 1 receptor antagonists induce human in-vitro adipogenesis through peroxisome proliferator-activated receptor-gamma activation. J Hypertens 2006; 24: 1809-1816.

12 Schupp M, Janke J, Clasen R, Unger T, Kintscher U. Angiotensin type 1 receptor blockers induce peroxisome proliferator-activated receptor-gamma activity. Circulation 2004; 109: 2054-2057.

13 Stangier J, Su CA, Roth W. Pharmacokinetics of orally and intravenously administered telmisartan in healthy young and elderly volunteers and in hypertensive patients. J Int Med Res 2000; 28: 149-167.

14 Honjo S, Nichi Y, Wada Y, Hamamoto Y, Koshiyama H. Possible beneficial effect of telmisartan on glycemic control in diabetic subjects. Diabetes Care 2005; 28: 498.

15 Michel MC, Bohner H, Koster J, Schafers R, Heemann U. Safety of telmisartan in patients with arterial hypertension : an open-label observational study. Drug Saf 2004; 27: 335-344.

16 Shimabukuro M, Tanaka H, Shimabukuro T. Effects of telmisartan on fat distribution in individuals with the metabolic syndrome. J Hypertens 2007; 25: 841-848.

17 Derosa G, Ragonesi PD, Mugellini A, Ciccarelli L, Fogari R. Effects of telmisartan compared with eprosartan on blood pressure control, glucose metabolism and lipid profile in hypertensive, type 2 diabetic patients: a randomized, double-blind, placebocontrolled 12-month study. Hypertens Res 2004; 27: 457-464.

18 Imai Y, Otsuka K, Kawano Y, Shimada K, Hayashi H, Tochikubo O, Miyakawa M, Fukiyama K. Japanese society of hypertension (JSH) guidelines for self-monitoring of blood pressure at home. Hypertens Res 2003; 26: 771-782.

19 Sakai H, Tsuchiya K, Nakayama C, Iwashima F, Izumiyama H, Doi M, Yoshimoto T, Tsujino M, Yamada S, Hirata Y. Improvement of endothelial dysfunction in acromegaly after transsphenoidal surgery. Endocr J 2008; 55: 853-859.

20 Tsuchiya K, Nakayama C, Iwashima F, Sakai H, Izumiyama H, Doi M, Hirata Y. Advanced endothelial dysfunction in diabetic patients with multiple risk factors; importance of insulin resistance. J Atheroscler Thromb 2007; 14: 303-309.

21 Dzau VJ. Theodore Cooper Lecture: tissue angiotensin and pathobiology of vascular disease: a unifying hypothesis. Hypertension 2001; 37: 1047-1052.

22 Pershadsingh HA. Treating the metabolic syndrome using angiotensin receptor antagonists that selectively modulate peroxisome proliferator-activated receptor-gamma. Int J Biochem Cell Biol 2006; 38: 766-781.

23 Miura Y, Yamamoto N, Tsunekawa S, Taguchi S, Eguchi Y, Ozaki N, Oiso Y. Replacement of valsartan and candesartan by telmisartan in hypertensive patients with type 2 diabetes: metabolic and antiatherogenic consequences. Diabetes Care 2005; 28: 757-758.

24 Perl S, Schmölzer I, Sourij H, Pressl H, Eder M, Zweiker R, Wascher TC. Telmisartan improves vascular function independently of metabolic and antihypertensive effects in hypertensive subjects with impaired glucose tolerance. Int J Cardiol 2010; 139: 289-296.

25 Vitale C, Mercuro G, Castiglioni C, Cornoldi A, Tulli A, Fini M, Volterrani M, Rosano GM. Metabolic effect of telmisartan and losartan in hypertensive patients with metabolic syndrome. Cardiovasc Diabetol 2005; 4: 6.

26 Yusuf S, Teo KK, Pogue J, Dyal L, Copland I, Schumacher H, Dagenais G, Sleight P, Anderson C. Telmisartan, ramipril, or both in patients at high risk for vascular events. $N$ Engl J Med 2008; 358: 1547-1559.
27 Benndorf RA, Appel D, Maas R, Schwedhelm E, Wenzel UO, Boger RH. Telmisartan improves endothelial function in patients with essential hypertension. J Cardiovasc Pharmacol 2007; 50: 367-371.

28 Ceriello A, Piconi L, Esposito K, Giugliano D. Telmisartan shows an equivalent effect of vitamin $C$ in further improving endothelial dysfunction after glycemia normalization in type 1 diabetes. Diabetes Care 2007; 30: 1694-1698.

29 Chujo D, Yagi K, Asano A, Muramoto H, Sakai S, Ohnishi A, Shintaku-Kubota M, Mabuchi H, Yamagishi M, Kobayashi J. Telmisartan treatment decreases visceral fat accumulation and improves serum levels of adiponectin and vascular inflammation markers in Japanese hypertensive patients. Hypertens Res 2007; 30: 1205-1210.

30 Delles C, Raff U, Mimran A, Fauvel JP, Ruilope LM, Schmieder RE. Effects of telmisartan and ramipril on adiponectin and blood pressure in patients with type 2 diabetes. Am J Hypertens 2008; 21: 1330-1336.

31 Makita S, Abiko A, Naganuma Y, Moriai Y, Nakamura M. Potential effects of angiotensin II receptor blockers on glucose tolerance and adiponectin levels in hypertensive patients. Cardiovasc Drugs Ther 2007; 21: 317-318.

32 Maeda N, Takahashi M, Funahashi T, Kihara S, Nishizawa H, Kishida K, Nagaretani H, Matsuda M, Komuro R, Ouchi N, Kuriyama H, Hotta K, Nakamura T, Shimomura I, Matsuzawa Y. PPARgamma ligands increase expression and plasma concentrations of adiponectin, an adipose-derived protein. Diabetes 2001; 50: 2094-2099.

33 Funahashi T, Matsuzawa Y. Hypoadiponectinemia: a common basis for diseases associated with overnutrition. Curr Atheroscler Rep 2006; 8: 433-438.

34 Tsuchiya K, Akaza I, Yoshimoto T, Hirata Y. Pioglitazone improves endothelial function with increased adiponectin and high-density lipoprotein cholesterol levels in type 2 diabetes. Endocr J 2009; 56: 691-698.

35 Torigoe M, Matsui H, Ogawa Y, Murakami H, Murakami R, Cheng XW, Numaguchi Y, Murohara T, Okumura K. Impact of the high-molecular-weight form of adiponectin on endothelial function in healthy young men. Clin Endocrinol (Oxf) 2007; 67: 276-281.

36 Hara K, Horikoshi M, Yamauchi T, Yago H, Miyazaki O, Ebinuma H, Imai Y, Nagai R, Kadowaki T. Measurement of the high-molecular weight form of adiponectin in plasma is useful for the prediction of insulin resistance and metabolic syndrome. Diabetes Care 2006; 29: 1357-1362.

37 Chen H, Montagnani M, Funahashi T, Shimomura I, Quon MJ. Adiponectin stimulates production of nitric oxide in vascular endothelial cells. J Biol Chem 2003; 278: $45021-45026$.

38 Nadar S, Blann AD, Lip GY. Antihypertensive therapy and endothelial function. Curr Pharm 2004; 10: 3607-3614.

39 Pasini AF, Garbin U, Stranieri C, Boccioletti V, Mozzini C, Manfro S, Pasini A Cominacini M, Cominacini L. Nebivolol treatment reduces serum levels of asymmetric dimethylarginine and improves endothelial dysfunction in essential hypertensive patients. Am J Hypertens 2008; 21: 1251-1257.

40 Yilmaz MI, Saglam M, Sonmez A, Caglar K, Cakir E, Kurt Y, Eyileten T, Tasar M, Acikel C, Oguz Y, Vural A, Yenicesu M. Improving proteinuria, endothelial functions and asymmetric dimethylarginine levels in chronic kidney disease: ramipril versus valsartan. Blood Purif 2007; 25: 327-335.

41 Makita S, Abiko A, Naganuma Y, Moriai Y, Nakamura M. Effects of telmisartan on adiponectin levels and body weight in hypertensive patients with glucose intolerance. Metabolism 2008; 57: 1473-1478. 